\title{
MEASUREMENT OF PRODUCTIVITY AND EFFICIENCY OF POTATO PRODUCTION IN TWO SELECTED AREAS OF BANGLADESH: A TRANSLOG STOCHASTIC FRONTIER ANALYSIS
}

\author{
A. Begum, M. F. Imam and M. A. Alam \\ Department of Agricultural Statistics, Bangladesh Agricultural University \\ Mymensingh-2202, Bangladesh
}

\begin{abstract}
Some partial analyses were used to determine the productivity of potato production. The per hectare potato production of the farmers of Lalmonirhat Sadar and Aditmari Upazila were 19897.88 and $21208.47 \mathrm{~kg}$ respectively. The benefit-cost ratio in Lalmonirhat Sadar and Aditmari Upazila were 1.52 and 1.56 respectively. The coefficient of farm size was positively significant in Aditmari in the inefficiency effect model, which meant large farmer was economically less efficient than small farmer. The sign of education was negative and significant, which indicates that inefficiency decreases with the increase of education in Aditmari Upazila. The economic efficiency varied from 81 to $99 \%$ at aggregate level, 97 to $99 \%$ in Lalmonirhat Sadar and 72 to $99 \%$ in Aditmari Upazila. The mean economic efficiencies were 98, 97 and 96\% for Lalmonirhat Sadar, Aditmari Upazila and at aggregate, level respectively. There appeared to be 2, 3 and $4 \%$ economic inefficiencies for Lalmonirhat Sadar, Aditmari and all regions, respectively. This indicates that the cost of production could be reduced on an average by $4 \%$ keeping the output constant at the aggregate level.
\end{abstract}

Key Words: Translog, Stochastic, Productivity, Efficiency

\section{INTRODUCTION}

Agriculture contributes 20.83 percent to the total gross domestic product (GDP) of Bangladesh (BBS, 2008). Though rice and wheat are main food crops, their production is not sufficient to meet the increasing requirements for the growing population in the country. In this regard, potato can play an important role as an alternative and a multipurpose food crop of Bangladesh. It is also an important cash crop for the farmers. It has the desirable characteristics of high yields, nutritious or delicious food and palatable in taste. It is one of the most important sources of carbohydrates and it contains an appreciable amount of vitamin B and C and some other materials (Thompson and Kelly, 1957).

In Bangladesh soil and climatic condition has offer high potential of potato production. It is the third largest food crop following rice and wheat. Bangladesh produces potato in about 0.30 million hectares of land with an average yield of 20.42 ton/ha in 2005-06 (BBS, 2008). Potato occupied the first position among all the vegetables in respect of area and total production. 
To earn the sustainable self-sufficiency in food for an increasing population, the diversified uses of potato as substitutes of food grain can play a major role. The role of potato bears upon the rate and structure of economic growth, rate of poverty nutrition for health, the trade balance and the fiscal position of the government. Although Bangladesh has achieved near self-sufficiency in food-grain production in the recent years, she cannot provide balanced diet to all her population. To solve the malnutrition problem of the country, emphasis should be given to produce more non-cereal crops like potato. In order to find out the potentials and possibilities of expansion in the acreage and production of the minor crops like potato, it is, therefore, important to examine the productivity and efficiency of farmers in producing potato. If farmers are found to be economically inefficient, production can be increased to large extent using the existing level of inputs and available technology. It can be done by increasing the productivity of inputs or by reallocating and combining them optimally. On the other hand, in case of efficient farmers, production can be increased by increasing cultivable area or substituting existing technology with more advanced technology. An appropriate and realistic agricultural policy is one of the most important instruments through which potato production can be increased but proper policy can be formulated only after the empirical measurement of the productivity and efficiency.

In the production efficiency arena, we are familiar with three types of efficiency namely technical, allocative and economics efficiencies. In this study we consider economic efficiency. Farrell (1957) proposed that economic or overall efficiency of a firm consists of two components technical efficiency, which reflects the ability of a firm to obtain maximal output from a given sets of inputs under certain production technology and allocative efficiency which reflects the ability of firm to use the inputs in optimal proportion given their respective prices. If a firm has achieved both technically and allocatively efficient levels of production, then the farm is economically efficient.

Economic relationships based on optimisation behaviour define efficient frontiers of minimum (e.g. cost) or maximum (e.g. production) attainment. Traditional econometric methods for estimating stochastic economic relationships have implicitly assumed that all economic agents are successful in reaching the efficient frontier. If, however, the economic agents are not equally efficient, then the average relationships estimated by ordinary least squares methods might not reflect the frontier relationships (Stevenson, 1980). Our purpose here is to develop a specification and estimation for a stochastic frontier model to estimate economic efficiency of the potato production in the selected area.

The objectives of this study, therefore, are (i) to measure the productivity and profitability of potato production in two selected areas of Bangladesh; (ii) to estimate the economic efficiencies of potato farmers; (iii) to identify factors which influence potato production; (v) to suggest some policies to increase productivity, profitability and efficiency of potato production. 
This paper has been organized in four section. In sections 2 data and methodology, specification of stochastic cost frontier and economic inefficiency effect model are described. Section 3 contains empirical results and discussions. Some conclusions are made in the final section.

\section{DATA SOURCE AND METHODOLOGY}

Two Upazilas namely: Aditmri and Lalmonirhat Sadar of Lalmonirhat district were selected purposely considering the relative importance of potato production. To collect the primary data it was not possible to include all the farmers under the study area because of limitation of time, money and personnel. For minimizing costs and time and achieving the ultimate objectives of the study 100 farmers were selected randomly from the study area. Probability sampling technique was adopted. At first a sampling frame of farmers was constructed with the help of village leaders and some other relevant people. Afterwards a stratified random sampling method was used to select the potato farmers for the study. The data were collected for the period January to March 2006. The sample was composed of small (below 1.00 hectare), medium (1.00-300 hectare) and large (above 3.00 hectare) farms respectively. Among the 100 potato farmers 50 small 30 medium and 20 large farmers were interviewed in this study.

\section{Model specification}

For this study, a Translog Stochastic Cost Frontier was used. The Cobb-Douglas form of production or cost function imposes certain restrictions, such as, the elasticity coefficients are constant, implying constant shares regardless of the input level or input cost and the elasticity of substitution among inputs is unity, whereas the translog production or cost function does not impose these restrictions upon the production or cost structure and it is a flexible functional form. Another advantage of translog stochastic frontier function is that with the help of this function we can estimate the effects of interactions of different complementary variables along with the individual effect of each variable on output or cost.

An explicit form of Translog Stochastic Frontier Cost Function (TSFCF) for potato production is presented below :

$\operatorname{lnc}=\alpha_{0}+\alpha_{1} \ln W+\alpha_{2} \ln \mathrm{P}_{\mathrm{s}}+\alpha_{3} \ln \mathrm{P}_{\mathrm{f}}+\alpha_{4} \ln \mathrm{P}_{\mathrm{m}}+\alpha_{5} \ln \mathrm{P}_{\mathrm{c}}+\alpha_{6} \ln \mathrm{R}_{\mathrm{l}}+\alpha_{7} \ln \mathrm{C}_{\mathrm{i}}+\alpha_{8} \ln \mathrm{Q}+$ $1 / 2 \beta_{11}(\ln W)^{2}+\beta_{12} \ln W \times \ln P_{s}+\beta_{13} \ln W \times \ln P_{f}+\beta_{14} \ln W \times \ln P_{m}+\beta_{15} \ln W \times \ln P_{c}+\beta_{16}$ $\ln W \times \ln R_{1}+\beta_{17} \ln W \times \ln C_{i}+\beta_{18} \ln W \times \ln Q+1 / 2 \beta_{22}\left(\ln P_{s}\right)^{2}+\beta_{23} \ln P_{s} \times \ln P_{f}+\beta_{24} \ln P_{s}$ $\times \ln P_{m}+\beta_{25} \ln P_{s} \times \ln P_{c}+\beta_{26} \ln P_{s} \times \ln R_{l}+\beta_{27} \ln P_{s} \times \ln C_{i}+\beta_{28} \ln P_{s} \times \ln Q+1 / 2 \beta_{33}(\ln$ $\left.\mathrm{P}_{\mathrm{f}}\right)^{2}+\beta_{34} \ln \mathrm{P}_{\mathrm{f}} \times \ln \mathrm{P}_{\mathrm{m}}+\beta_{35} \ln \mathrm{P}_{\mathrm{f}} \times \ln \mathrm{P}_{\mathrm{c}}+\beta_{36} \ln \mathrm{P}_{\mathrm{f}} \times \ln \mathrm{R}_{\mathrm{l}}+\beta_{37} \ln \mathrm{P}_{\mathrm{f}} \times \ln \mathrm{C}_{\mathrm{i}}+\beta_{38} \ln \mathrm{P}_{\mathrm{f}} \times \ln \mathrm{Q}$ $+1 / 2 \beta_{44}\left(\ln P_{m}\right)^{2}+\beta_{45} \ln P_{m} \times \ln P_{c}+\beta_{46} \ln P_{m} \times \ln R_{l}+\beta_{47} \ln P_{m} \times \ln C_{i}+\beta_{48} \ln P_{m} \times \ln Q+$ $1 / 2 \beta_{55}\left(\ln P_{c}\right)^{2}+\beta_{56} \ln P_{c} \times \ln R_{1}+\beta_{57} \ln P_{c} \times \ln C_{i}+\beta_{58} \ln P_{c} \times \ln Q+1 / 2 \beta_{66}\left(\ln R_{l}\right)^{2}+\beta_{67} \ln R_{l}$ $\times \ln C_{i}+\beta_{68} \ln R_{1} \times \ln Q+1 / 2 \beta_{77}\left(\ln C_{i}\right)^{2}+\beta_{78} \ln C_{i} \times \ln Q+1 / 2 \beta_{88}(\ln Q)^{2}+\beta_{9} \ln ($ Age $)+\beta_{10}$ EXPERIENCE $+\beta_{19}$ EDU $+\beta_{20}$ EXT. $+\mathrm{V}+\mathrm{U}$ 
Where, $\mathrm{W}=$ Human labour price, $\mathrm{Ps}=$ Seed price, $\mathrm{P}_{\mathrm{f}}=$ Fertilizer price, $\mathrm{P}_{\mathrm{m}}=$ Manure price $P_{c}=$ Power tiller cost, $R_{l}=$ Per hectare rent of land, $C_{i}=$ Per hectare irrigation cost $\mathrm{Q}=$ Output

and Age is age of potato farmer, EXPERIENCE is experience of potato farmer, EDU is education of potato farmer and EXT. is extension service.

$\mathrm{U}$ is a non-negative cost (or economic) inefficiency effect, which is assumed to have a half normal distribution and $\mathrm{V}$ is random variable, which is assumed to be independently and normally distributed with $\mathrm{N}\left(0, \sigma_{\mathrm{v}}{ }^{2}\right)$. $\mathrm{U}$ is added in the cost frontier, instead of being subtracted, as in the case of production frontier. This is because the cost frontier represents minimum costs, whereas the production represents maximum output. The $U$ provides information on the level of the cost efficiency or overall economic efficiency (EE).

The model for the economic inefficiency effects in the stochastic frontier of equation (1) is defined as

$\mathrm{U}_{\mathrm{i}}=\delta_{0}+\delta_{1}$ Farmsz Age $+\delta_{2}$ Age $+\delta_{3}$ Experience $+\delta_{4}$ Edu $+\delta_{5}$ Contact $+\mathrm{W}_{\mathrm{i}} \ldots$ (2)

Where, Age, Edu and Experience defined earlier, Contact represents extension contact by the extension agents to the farmers.

Farmsz represents farm size and the $\mathrm{W}_{\mathrm{i}}$ are unobservable random variables, which are assumed to be independently distributed with a positive half normal distribution.

The $\beta$ - and $\delta$-coefficients are unknown parameters to be estimated, together with the variance parameters which are expressed in terms of -

$$
\begin{gathered}
\sigma^{2}=\sigma_{u}^{2}+\sigma_{v}^{2} \\
\gamma=\sigma_{u}^{2} / \sigma^{2}
\end{gathered}
$$

Where, the $\gamma$-parameter has a value between zero and one.

The model for the inefficiency effects can only be estimated if the inefficiency effects are stochastic and have a particular distributional specification. Hence there is interest to test the null hypotheses that the efficiency effects are not present, $\mathrm{H}_{0}: \gamma=\delta_{0}=\delta_{1}=\delta_{2}=\delta_{3}=\delta_{4}=$ $\delta_{5}=0$; the inefficiency effects are not stochastic, $\mathrm{H}_{0}=\gamma=0$; and the coefficients of the variables in the model for the inefficiency effects are zero, $\mathrm{H}_{0}=\delta_{1}=\delta_{2}=\ldots=\delta_{5}=0$. These and other null hypotheses of interest will be tested using the generalized likelihood-ratio test and t-test. Under the null hypotheses, $\mathrm{H}_{0}=\gamma=0$, the model is equivalent to the traditional average response function without the economic inefficiency effect, $\mathrm{U}_{\mathrm{i}}$. The test statistic is calculated as

$$
\mathrm{LR}=-2\left\{\ln \left[\mathrm{L}\left(\mathrm{H}_{0}\right) / \mathrm{L}\left(\mathrm{H}_{1}\right)\right]\right\}=-2\left\{\ln \left[\mathrm{L}\left(\mathrm{H}_{0}\right)\right]-\ln \left[\mathrm{L}\left(\mathrm{H}_{\mathrm{i}}\right)\right]\right\}
$$

Where, $\mathrm{L}\left(\mathrm{H}_{0}\right)$ and $\mathrm{L}\left(\mathrm{H}_{\mathrm{i}}\right)$ are the values of the likelihood function under the null and alternative hypotheses, $\mathrm{H}_{0}$ and $\mathrm{H}_{1}$ respectively. 
For this study, the parameters of the Translog Stochastic frontier cost function model are estimated by the maximum likelihood method, using the computer program, FRONTIER Version 4.1 developed by Coelli (1996a) together with region-specific economic efficiencies and mean economic efficiency for the farms involved and also inefficiency effect model.

\section{RESULTS AND DISCUSSION}

Output, net return and the benefit-cost ratio expressed per unit of a single input category are partial measures of productivity. Physical output or value of output per hectare and other similar measures may be considered a measure of average performance in the productivity scale (Britton and Hill, 1975). Table 1 reveals that the productivity is higher in Aditmari Upazila (21208.47 kg) than in Lalmonirhat Sadar Upazila (19897.88 kg) and there is no significant difference in per hectare net return between the farmers of Aditmari (Tk. 21867.41) and Lalmonirhat Sadar Upazila (Tk. 19340.26).

Table 1. Per hectare production (output), net return and benefit-cost ratio of potato in the selected areas

\begin{tabular}{lcc|c}
\hline Regions & $\begin{array}{c}\text { Per hectare potato } \\
\text { production }(\mathrm{kg})\end{array}$ & Net return (in Tk.) & Benefit-cost ratio \\
\hline Lalmonirhat Sadar & 19897.88 & 19340.28 & 1.52 \\
& $(548.78)$ & $(4520.05)$ & $(0.10)$ \\
Aditmari & 21208.47 & 21867.41 & 1.56 \\
& $(577.78)$ & $(10580.85)$ & $(0.09)$ \\
Total & 20553.18 & 20603.84 & 1.54 \\
& $(401.61)$ & $(6000.90)$ & $(0.09)$ \\
Z-value & 1.640 & 0.697 & 0.220 \\
\hline
\end{tabular}

Source : Field Survey, 2006

Figures within parentheses indicate standard error of the estimates

Table 2 presents simultaneous estimation of the translog stochastic cost frontier and economic inefficiency effect model for potato production. Although the simultaneous estimation procedure has simultaneous -equation bias, it is also important to identify the factors, which influence the technical or economic inefficiency of farmers. Kumbhakar, Ghose and Mcguckin (1991), Reifschneider and Stevension (1991), Haung and Lui (1994) and Battese and Coelli (1995) specify stochastic frontiers and models for the technical inefficiency effects and simultaneously estimate all the parameters involved. This one stage approach is less objectionable from a statistical point of view and is expected to lead to more efficient inference with respect to the parameters involved. Estimation procedure of economic efficiency is the same as that of technical efficiency where the former is estimated from the cost frontier and the latter is estimated from the production frontier. 
Table 2. Maximum likelihood (ML) estimates of translog stochastic cost frontier and economic inefficiency model

\begin{tabular}{|c|c|c|c|c|}
\hline \multirow[t]{2}{*}{ Variables } & \multirow[t]{2}{*}{ Parameters } & \multirow{2}{*}{$\begin{array}{l}\text { Aggregate } \\
\text { level }\end{array}$} & \multicolumn{2}{|c|}{ Region-specific } \\
\hline & & & $\begin{array}{l}\text { Lalmonirhat } \\
\text { Sadar }\end{array}$ & Aditmari \\
\hline Intercept & $\alpha_{0}$ & $\begin{array}{c}-22.8772^{* *} \\
(1.0000)\end{array}$ & $\begin{array}{l}8.1466^{* *} \\
(1.0000)\end{array}$ & $\begin{array}{c}141.2685^{* *} \\
(0.9802)\end{array}$ \\
\hline Human labour price & $\alpha_{1}$ & $\begin{array}{l}-0.8013 \\
(1.0000)\end{array}$ & $\begin{array}{l}-4.6245^{* *} \\
(1.0000)\end{array}$ & $\begin{array}{c}18.1289 * * \\
(0.9535)\end{array}$ \\
\hline Seed price & $\alpha_{2}$ & $\begin{array}{l}-2.8461^{* *} \\
(1.0000)\end{array}$ & $\begin{array}{l}-4.1154^{* *} \\
(1.0000)\end{array}$ & $\begin{array}{c}-53.5163^{* *} \\
(0.8467)\end{array}$ \\
\hline Fertilizer price & $\alpha_{3}$ & $\begin{array}{l}3.8344^{* *} \\
(1.0000)\end{array}$ & $\begin{array}{l}-2.1985^{*} \\
(1.0000)\end{array}$ & $\begin{array}{c}-23.8226^{* *} \\
(0.9770)\end{array}$ \\
\hline Manure price & $\alpha_{4}$ & $\begin{array}{l}4.7773^{* *} \\
(1.0000)\end{array}$ & $\begin{array}{l}-0.6929 \\
(1.0000)\end{array}$ & $\begin{array}{l}-41.9164^{* *} \\
(0.9738)\end{array}$ \\
\hline Power tiller cost & $\alpha_{5}$ & $\begin{array}{l}-8.5474^{* *} \\
(1.0000)\end{array}$ & $\begin{array}{c}-12.1843^{* *} \\
(1.0000)\end{array}$ & $\begin{array}{c}-25.4835^{* *} \\
(0.9295)\end{array}$ \\
\hline Per hectare rent of land & $\alpha_{6}$ & $\begin{array}{l}4.9352^{* *} \\
(1.0000)\end{array}$ & $\begin{array}{c}1.8726 \\
(1.0000)\end{array}$ & $\begin{array}{c}-34.2258^{* *} \\
(0.4729)\end{array}$ \\
\hline Per hectare irrigation cost & $\alpha_{7}$ & $\begin{array}{l}4 . .4854^{* *} \\
(1.0000)\end{array}$ & $\begin{array}{l}2.3474^{*} \\
(1.0000)\end{array}$ & $\begin{array}{c}-63.5938 * * \\
(0.9743)\end{array}$ \\
\hline Output & $\alpha_{8}$ & $\begin{array}{l}9.1635^{* *} \\
(1.0000)\end{array}$ & $\begin{array}{c}1.0726 \\
(1.0000)\end{array}$ & $\begin{array}{c}68.5565^{* *} \\
(0.9555)\end{array}$ \\
\hline $\begin{array}{l}\text { Human labour price } \times \text { Human } \\
\text { labour price }\end{array}$ & $\beta_{11}$ & $\begin{array}{c}0.0091 \\
(1.0000)\end{array}$ & $\begin{array}{l}-0.1455 \\
(1.0000)\end{array}$ & $\begin{array}{c}-1.0172^{* *} \\
(0.1586)\end{array}$ \\
\hline H. labour price $\times$ Seed price & $\beta_{12}$ & $\begin{array}{l}-0.0814 \\
(1.0000)\end{array}$ & $\begin{array}{l}-0.1269 \\
(1.0000)\end{array}$ & $\begin{array}{c}-4.0599 * * \\
(0.0968)\end{array}$ \\
\hline H. labour price $\times$ Fertilizer price & $\beta_{13}$ & $\begin{array}{c}0.0275 \\
(1.0000)\end{array}$ & $\begin{array}{c}0.0510 \\
(1.0000)\end{array}$ & $\begin{array}{l}5.2252^{* *} \\
(0.1117)\end{array}$ \\
\hline H. labour price $\times$ Manure price & $\beta_{14}$ & $\begin{array}{l}-0.1175 \\
(1.0000)\end{array}$ & $\begin{array}{l}-0.0959 \\
(1.0000)\end{array}$ & $\begin{array}{l}-1.3352 \\
(0.7174)\end{array}$ \\
\hline H. labour price $\times$ Power tiller cost & $\beta_{15}$ & $\begin{array}{c}0.1688 \\
(1.0000)\end{array}$ & $\begin{array}{c}0.2485 \\
(1.0000)\end{array}$ & $\begin{array}{c}0.1516 \\
(0.1368)\end{array}$ \\
\hline H. labour price $\times$ P.h. rent of land & $\beta_{16}$ & $\begin{array}{c}0.0551 \\
(1.0000)\end{array}$ & $\begin{array}{c}0.5863 \\
(1.0000)\end{array}$ & $\begin{array}{c}-3.5996^{* *} \\
(0.1084)\end{array}$ \\
\hline H. labour price $\times$ P.h. irrigation cost & $\beta_{17}$ & $\begin{array}{c}0.0972 \\
(1.0000)\end{array}$ & $\begin{array}{c}0.0759 \\
(1.0000)\end{array}$ & $\begin{array}{l}2.8276^{* *} \\
(0.0682)\end{array}$ \\
\hline H. labour price $\times$ Output & $\beta_{18}$ & $\begin{array}{l}-0.0255 \\
(1.0000)\end{array}$ & $\begin{array}{l}-0.0458 \\
(1.0000)\end{array}$ & $\begin{array}{c}-0.0679 \\
(0.126 .8)\end{array}$ \\
\hline
\end{tabular}




\begin{tabular}{|c|c|c|c|c|}
\hline \multirow[t]{2}{*}{ Variables } & \multirow[t]{2}{*}{ Parameters } & \multirow{2}{*}{$\begin{array}{l}\text { Aggregate } \\
\text { level }\end{array}$} & \multicolumn{2}{|c|}{ Region-specific } \\
\hline & & & $\begin{array}{l}\text { Lalmonirhat } \\
\text { Sadar }\end{array}$ & Aditmari \\
\hline Seed price $\times$ Seed price & $\beta_{22}$ & $\begin{array}{c}0.2857 \\
(1.0000)\end{array}$ & $\begin{array}{c}0.4511 \\
(1.0000)\end{array}$ & $\begin{array}{l}2.7052^{* *} \\
(0.1374)\end{array}$ \\
\hline Seed price $\times$ Fertilizer price & $\beta_{23}$ & $\begin{array}{c}0.1144 \\
(1.0000)\end{array}$ & $\begin{array}{c}0.3537 \\
(1.0000)\end{array}$ & $\begin{array}{l}0.4585^{* *} \\
(0.1583)\end{array}$ \\
\hline Seed price $\times$ Manure price & $\beta_{24}$ & $\begin{array}{c}0.0380 \\
(1.0000)\end{array}$ & $\begin{array}{l}-0.1623 \\
(1.0000)\end{array}$ & $\begin{array}{l}-0.1569 \\
(0.0819)\end{array}$ \\
\hline Seed price $\times$ Power tiller cost & $\beta_{25}$ & $\begin{array}{l}-0.0662 \\
(1.0000)\end{array}$ & $\begin{array}{l}-0.0948 \\
(1.0000)\end{array}$ & $\begin{array}{l}2.5287^{* *} \\
(0.0952)\end{array}$ \\
\hline Seed price $\times$ P.h. rent of land & $\beta_{26}$ & $\begin{array}{c}0.3352 \\
(1.0000)\end{array}$ & $\begin{array}{c}0.3388 \\
(1.0000)\end{array}$ & $\begin{array}{l}9.5684^{* *} \\
(0.1249)\end{array}$ \\
\hline Seed price $\times$ P.h. irrigation cost & $\beta_{27}$ & $\begin{array}{l}-0.0657 \\
(1.0000)\end{array}$ & $\begin{array}{c}0.1311 \\
(1.0000)\end{array}$ & $\begin{array}{c}-3.8361^{* *} \\
(0.0724)\end{array}$ \\
\hline Seed price $\times$ Output & $\beta_{28}$ & $\begin{array}{l}-0.2396 \\
(1.0000)\end{array}$ & $\begin{array}{l}-0.4211 \\
(1.0000)\end{array}$ & $\begin{array}{l}-0.1779 \\
(0.1122)\end{array}$ \\
\hline Fertilizer price $\times$ Fertilizer price & $\beta_{33}$ & $\begin{array}{c}0.1062 \\
(1.0000)\end{array}$ & $\begin{array}{c}0.1873 \\
(1.0000)\end{array}$ & $\begin{array}{c}-0.6448^{* *} \\
(0.1044)\end{array}$ \\
\hline Fertilizer price $\times$ Manure price & $\beta_{34}$ & $\begin{array}{c}0.0570 \\
(1.0000)\end{array}$ & $\begin{array}{l}-0.1889 \\
(1.0000)\end{array}$ & $\begin{array}{c}-2.1452 * * \\
(0.0939)\end{array}$ \\
\hline Fertilizer price $\times$ Power tiller cost & $\beta_{35}$ & $\begin{array}{l}-0.2832 \\
(1.0000)\end{array}$ & $\begin{array}{l}-0.2446 \\
(1.0000)\end{array}$ & $\begin{array}{c}-3.8229 * * \\
(0.1791)\end{array}$ \\
\hline Fertilizer price $\times$ P.h. rent of land & $\beta_{36}$ & $\begin{array}{l}-0.4672 \\
(1.0000)\end{array}$ & $\begin{array}{c}0.3021 \\
(1.0000)\end{array}$ & $\begin{array}{l}1.4586^{* *} \\
(0.1203)\end{array}$ \\
\hline Fertilizer price $\times$ P.h. irrigation cost & $\beta_{37}$ & $\begin{array}{l}-0.0356 \\
(1.0000)\end{array}$ & $\begin{array}{l}-0.2944 \\
(1.0000)\end{array}$ & $\begin{array}{c}0.2345 \\
(0.3996)\end{array}$ \\
\hline Fertilizer price $\times$ Output & $\beta_{38}$ & $\begin{array}{c}0.0448 \\
(1.0000)\end{array}$ & $\begin{array}{l}-0.0199 \\
(1.0000)\end{array}$ & $\begin{array}{c}0.1483 \\
(0.1632)\end{array}$ \\
\hline Manure price $\times$ Manure price & $\beta_{44}$ & $\begin{array}{l}-0.2420 \\
(1.0000)\end{array}$ & $\begin{array}{c}0.0832 \\
(1.0000)\end{array}$ & $\begin{array}{c}-5.9980 * * \\
(0.0999)\end{array}$ \\
\hline Manure price $\times$ Power tiller cost & $\beta_{45}$ & $\begin{array}{c}0.1371 \\
(1.0000)\end{array}$ & $\begin{array}{c}0.2513 \\
(1.0000)\end{array}$ & $\begin{array}{l}3.8495^{\star *} \\
(0.1288)\end{array}$ \\
\hline Manure price $\times$ P.h. rent of land & $\beta_{46}$ & $\begin{array}{l}-0.4214 \\
(1.0000)\end{array}$ & $\begin{array}{c}0.2257 \\
(1.0000)\end{array}$ & $\begin{array}{l}6.1344^{* *} \\
(0.1185)\end{array}$ \\
\hline Manure price $\times$ P.h. irrigation cost & $\beta_{47}$ & $\begin{array}{l}-0.1162 \\
(1.0000)\end{array}$ & $\begin{array}{c}0.0061 \\
(1.0000)\end{array}$ & $\begin{array}{l}-1.3320^{*} \\
(0.5413)\end{array}$ \\
\hline Manure price $\times$ Output & $\beta_{48}$ & $\begin{array}{c}0.1127 \\
(1.0000)\end{array}$ & $\begin{array}{l}-0.0101 \\
(1.0000)\end{array}$ & $\begin{array}{l}4.6322^{* *} \\
(0.1279)\end{array}$ \\
\hline
\end{tabular}




\begin{tabular}{|c|c|c|c|c|}
\hline \multirow[t]{2}{*}{ Variables } & \multirow[t]{2}{*}{ Parameters } & \multirow{2}{*}{$\begin{array}{l}\text { Aggregate } \\
\text { level }\end{array}$} & \multicolumn{2}{|c|}{ Region-specific } \\
\hline & & & $\begin{array}{l}\text { Lalmonirhat } \\
\text { Sadar }\end{array}$ & Aditmari \\
\hline Power tiller cost $\times$ Power tiller cost & $\beta_{55}$ & $\begin{array}{c}0.2141 \\
(1.0000)\end{array}$ & $\begin{array}{l}-0.1073 \\
(1.0000)\end{array}$ & $\begin{array}{l}6.1072 * * \\
(0.1907)\end{array}$ \\
\hline Power tiller cost $\times$ P.h. rent of land & $\beta_{56}$ & $\begin{array}{c}0.9170 \\
(1.0000)\end{array}$ & $\begin{array}{c}0.1487 \\
(1.0000)\end{array}$ & $\begin{array}{l}3.2426^{* *} \\
(0.1014)\end{array}$ \\
\hline $\begin{array}{l}\text { Power tiller cost } \times \text { P.h. irrigation } \\
\text { cost }\end{array}$ & $\beta_{57}$ & $\begin{array}{c}0.0791 \\
(1.0000)\end{array}$ & $\begin{array}{l}-0.2040 \\
(1.0000)\end{array}$ & $\begin{array}{l}0.8608^{* *} \\
(0.0419)\end{array}$ \\
\hline Power tiller cost $\times$ Output & $\beta_{58}$ & $\begin{array}{l}-0.1733 \\
(1.0000)\end{array}$ & $\begin{array}{l}-0.0351 \\
(1.0000)\end{array}$ & $\begin{array}{l}-9.8151^{* *} \\
(0.2462)\end{array}$ \\
\hline P.h. rent of land $\times$ P.h. rent of land & $\beta_{66}$ & $\begin{array}{l}-0.8808 \\
(1.0000)\end{array}$ & $\begin{array}{l}-0.2097 \\
(1.0000)\end{array}$ & $\begin{array}{l}3.7930 * * \\
(0.0526)\end{array}$ \\
\hline $\begin{array}{l}\text { P.h. rent of land } \times \text { P.h. irrigation } \\
\text { cost }\end{array}$ & $\beta_{67}$ & $\begin{array}{l}-0.5536 \\
(1.0000)\end{array}$ & $\begin{array}{l}-0.3786 \\
(1.0000)\end{array}$ & $\begin{array}{l}6.9491^{* *} \\
(0.1204)\end{array}$ \\
\hline P.h. rent of land $\times$ Output & $\beta_{68}$ & $\begin{array}{l}-0.1981 \\
(1.0000)\end{array}$ & $\begin{array}{l}-0.5450 \\
(1.0000)\end{array}$ & $\begin{array}{r}-10.1185^{* *} \\
(0.1381)\end{array}$ \\
\hline $\begin{array}{l}\text { P.h. irrigation cost } \times \text { P.h. irrigation } \\
\text { cost }\end{array}$ & $\beta_{77}$ & $\begin{array}{c}0.0843 \\
(1.0000)\end{array}$ & $\begin{array}{c}0.0831 \\
(1.0000)\end{array}$ & $\begin{array}{l}0.8419 * * \\
(0.0610)\end{array}$ \\
\hline P.h. irrigation cost $\times$ Output & $\beta_{78}$ & $\begin{array}{c}0.0535 \\
(1.0000)\end{array}$ & $\begin{array}{c}0.3021 \\
(1.0000)\end{array}$ & $\begin{array}{l}1.0012^{* *} \\
(0.0512)\end{array}$ \\
\hline Output $\times$ Output & $\beta_{88}$ & $\begin{array}{c}0.3319 \\
(1.0000)\end{array}$ & $\begin{array}{c}0.7065 \\
(1.0000)\end{array}$ & $\begin{array}{l}7.1289 * * \\
(0.2465)\end{array}$ \\
\hline Age of farm operator & $\beta_{9}$ & $\begin{array}{c}0.0523 \\
(1.0000)\end{array}$ & $\begin{array}{c}0.0731 \\
(1.0000)\end{array}$ & $\begin{array}{l}0.4972^{* *} \\
(0.0341)\end{array}$ \\
\hline Farming Experience & $\beta_{10}$ & $\begin{array}{l}-0.0103 \\
(1.0000)\end{array}$ & $\begin{array}{l}-0.0286 \\
(1.0000)\end{array}$ & $\begin{array}{l}0.1544^{* *} \\
(0.0100)\end{array}$ \\
\hline Education & $\beta_{19}$ & $\begin{array}{l}-0.0011 \\
(1.0000)\end{array}$ & $\begin{array}{l}-0.0027 \\
(1.0000)\end{array}$ & $\begin{array}{l}0.1075^{* *} \\
(0.0018)\end{array}$ \\
\hline Extension contact & $\beta_{20}$ & $\begin{array}{l}-0.0032 \\
(1.0000)\end{array}$ & $\begin{array}{l}-0.0097 \\
(1.0000)\end{array}$ & $\begin{array}{c}-0.4888^{* *} \\
(0.0092)\end{array}$ \\
\hline $\begin{array}{l}\text { Inefficiency effect model: } \\
\text { Intercept }\end{array}$ & $\delta_{0}$ & $\begin{array}{l}0.00001 \\
(1.0000)\end{array}$ & $\begin{array}{l}0.00001 \\
(1.0000)\end{array}$ & $\begin{array}{l}-3.3076^{* *} \\
(1.0295)\end{array}$ \\
\hline Farm size & $\delta_{1}$ & $\begin{array}{l}0.00001 \\
(1.0000)\end{array}$ & $\begin{array}{l}0.00001 \\
(1.0000)\end{array}$ & $\begin{array}{l}0.0268^{*} \\
(0.0123)\end{array}$ \\
\hline Age & $\delta_{2}$ & $\begin{array}{l}0.00001 \\
(1.0000)\end{array}$ & $\begin{array}{l}0.00001 \\
(1.0000)\end{array}$ & $\begin{array}{l}-0.1765 \\
(0.3073)\end{array}$ \\
\hline Farming experience & $\delta_{3}$ & $\begin{array}{l}0.00001 \\
(1.0000)\end{array}$ & $\begin{array}{l}0.00001 \\
(1.0000)\end{array}$ & $\begin{array}{c}0.0442 \\
(0.0754)\end{array}$ \\
\hline
\end{tabular}




\begin{tabular}{|c|c|c|c|c|}
\hline \multirow[t]{2}{*}{ Variables } & \multirow[t]{2}{*}{ Parameters } & \multirow{2}{*}{$\begin{array}{l}\text { Aggregate } \\
\text { level }\end{array}$} & \multicolumn{2}{|c|}{ Region-specific } \\
\hline & & & $\begin{array}{l}\text { Lalmonirhat } \\
\text { Sadar }\end{array}$ & Aditmari \\
\hline Education & $\delta_{4}$ & $\begin{array}{l}0.00001 \\
(1.0000)\end{array}$ & $\begin{array}{l}0.00001 \\
(1.0000)\end{array}$ & $\begin{array}{c}-0.0304^{* *} \\
(0.0086)\end{array}$ \\
\hline Extension contact & $\delta_{5}$ & $\begin{array}{l}0.00001 \\
(1.0000)\end{array}$ & $\begin{array}{l}0.00001 \\
(1.0000)\end{array}$ & $\begin{array}{l}-0.0041 \\
(0.0503)\end{array}$ \\
\hline \multirow[t]{2}{*}{ Variance parameters } & $\sigma^{2}$ & $\begin{array}{c}0.0036 \\
(1.0000)\end{array}$ & $\begin{array}{c}0.000005 \\
(1.0000)\end{array}$ & $\begin{array}{l}0.2213^{* *} \\
(0.0313)\end{array}$ \\
\hline & $\gamma$ & $\begin{array}{c}0.8000 \\
(1.0000)\end{array}$ & $\begin{array}{c}0.3700 \\
(1.0000)\end{array}$ & $\begin{array}{l}0.9999 * * \\
(0.00001)\end{array}$ \\
\hline Log likelihood function & & 180.3375 & 239.3612 & 103.3092 \\
\hline
\end{tabular}

Source: Own estimation

Figures within parentheses indicate asymptotic standard error

* and ** indicate significance at 0.05 and 0.01 probability level, respectively

In aggregate model the coefficients of fertilizer price, manure price, per hectare rent of land, per hectare irrigation cost and output are found to be positive and significant (significant at 1\% level) in the translog stochastic cost frontier. That means, fertilizer price, manure price, per hectare rent of land, per hectare irrigation cost and output have significantly positive contribution on the increase of cost of production of potato. However, the coefficients of seed price and power tiller cost are negative and significant in the cost frontier, which means that cost of potato production decreased with the increase in seed price and power tiller cost. The coefficient of age of farmers has positive but insignificant impact on the cost frontier. The coefficient of experience, education and extension contact are found to have negative but no significant impact on the cost frontier.

In the economic inefficiency effect model, it was observed that the coefficients of farm size, age and farming experience have no significant impact upon the inefficiency effects for potato production. Education and extension contact are found to have no impact on the production of potato. Bravo-Ureta and Rieger (1991) found the same result of extension contact. The generalized likelihood-ratio (LR) test statistic calculated from the model is 10.62, which is insignificant. This means that, there is no significant economic inefficiency effect in the production of potato.

In the region specific model, in Lalmonirhat Sadar Upazila, the coefficient of per hectare irrigation cost is found to be significant with positive signs, whereas the coefficients of human labour price, seed price, fertilizer price and power tiller cost are found to negatively significant in the cost frontier. Significantly positive coefficient of per hectare irrigation cost reveals that cost of potato production increases with the increase in per hectare irrigation cost. On the other hand, cost of potato production significantly decreases with the increase in human labour price, seed price, fertilizer price and power 
tiller cost separately. In the inefficiency effect model, we observe that there are no economic inefficiency effects.

In Aditmari Upazila, the coefficients of human labour price and output, squares of seed price, power tiller cost, per hectare rent of land, per hectare irrigation cost and output, interactions of human labour price \& per hectare irrigation cost, human labour price \& fertilizer price, seed price \& fertilizer price, seed price \& power tiller cost, seed price\& per hectare rent of land, fertilizer price \& per hectare rent of land, manure price \& power tiller cost, manure price \& per hectare rent of land, manure price \& output, power tiller price \& per hectare rent of land, power tiller cost \& per hectare irrigation cost, per hectare rent of land \& per hectare irrigation cost, per hectare irrigation cost \& output, age, experience and education are found to be positive and significant, whereas the coefficients of seed price, fertilizer price, manure price, power tiller cost, per hectare rent of land and per hectare irrigation cost, squares of human labour price, fertilizer price and manure price, interactions of human labour price \& seed price, human labour price \& per hectare rent of land, seed price \& per hectare irrigation cost, fertilizer price \& manure price, fertilizer price\& power tiller cost, manure price \& per hectare irrigation cost, power tiller cost \& output, per hectare rent of land \& output, extension contact are negative and significant. In the economic inefficiency effect model, the coefficient of farm size is positively significant (significant at $5 \%$ level) while the coefficient of education (years of schooling) is negatively significant (significant at $1 \%$ level). Significantly positive coefficient of farm size indicates that inefficiency increases with the increase in farm size, i.e., large farmers are economically less efficient than small farmers. Parikh et al. (1995) found the same result concerning farm size while studying economic efficiency in Pakistan agriculture. They said that small farmers seemed to be more efficient than the large farms in the region. Negatively significant coefficient of education implies that economic inefficiency decreases with the increase in education (years of schooling), that means educated farmers are economically more efficient than non educated or less educated farmers. Rahman (2002) found the same result in the production of rice in Bangladesh.

The variance ratio parameter $\gamma$ associated with the variances in the stochastic frontier is significantly positive for potato production in Aditmari Upazila, but insignificant in Lalmonirhat Sadar Upazila. It indicates that there are significant economic inefficiency effects in potato production in Aditmari Upazila, but there is no economic inefficiency effect in Lalmonirhat Sadar Upazila.

Table 3 shows the frequency distribution of economic efficiency estimate from translog stochastic cost frontier from the aggregate model. It reveals that the economic efficiency varies from $81 \%$ to $99 \%$. The maximum economic efficiency has been observed to be in the range of $95-97 \%$. The mean economic efficiency has been estimated from the translog stochastic cost frontier is $96 \%$. There appears to be $4 \%$ economic inefficiency at aggregate level for potato production. This implies that the cost of production can be reduced on average by $4 \%$ keeping the output constant. The region-specific economic efficiency 
varies from $97 \%$ to $99 \%$ and $72 \%$ to $99 \%$ for Lalmonirhat Sadar and Aditmari Upazila, respectively.

Table 3. Frequency distribution of economic efficiency estimates from translog stochastic cost frontier at aggregate level

\begin{tabular}{l|c}
\hline Efficiency level (\%) & Economic efficiency \\
\hline $80-82$ & $1(1.00)$ \\
$83-85$ & 0 \\
$86-88$ & $2(2.00)$ \\
$89-91$ & $3(3.00)$ \\
$92-94$ & $13(13.00)$ \\
$95-97$ & $62(62.00)$ \\
$98-100$ & $19(19.00)$ \\
Total number of farms & $100(100)$ \\
Mean efficiency & 96 \\
Maximum efficiency & 99 \\
Minimum efficiency & 81 \\
\hline
\end{tabular}

Source: Own estimation

Figures within parentheses indicate percentage

\section{Test of hypothesis}

We have already tested different coefficients on the translog stochastic cost frontier and economic inefficiency effect models with the help of $t$-test. Here we are going to test the coefficients of region specific variables on the economic inefficiency effect models using the generalized likelihood-ratio statistic LR. Coelli (1995) suggested that the one-sided generalized likelihood-ratio test should be performed when ML estimation is involved because this test has the correct size (i.e., probability of a Type I error). We were interested in testing the null hypotheses that the inefficiency effects were not present. In other words, the null hypothesis is that there are no economic inefficiency effects in the model.

That is, $\mathrm{H}_{0}: \gamma=\delta_{0}=\delta_{1}=\ldots=\delta_{5}=0$.

Table 4 reveals that there are no significant economic inefficiency effects in the production of potato in aggregate model since the null hypothesis is accepted. For regionspecific efficiency measures, there is no inefficiency effect in Lalmonirhat Sadar region, since the null hypothesis is accepted but economic inefficiency effects are found to be significant in Aditmari region, since the null hypothesis is rejected. 
Table 4. Test of hypothesis for coefficients of the explanatory variables for the economic inefficiency effects in the translog stochastic cost frontier

\begin{tabular}{lcc|c|c}
\hline \multicolumn{1}{c}{ Null hypothesis } & Log-likelihood value & Test statistic LR & Critical value & Decision \\
\hline $\begin{array}{l}\mathrm{H}_{0}: \gamma=\delta_{0}=\delta_{1}=\ldots=\delta_{5}=0 \\
\text { Aggregate level }\end{array}$ & 180.3375 & 10.6182 & 12.02 & Accepted \\
Region-specific : & & & & \\
Lalmonirhat Sadar & 239.3612 & 0.0794 & 12.02 & Accepted \\
Aditmari & 103.3092 & 371.3572 & 12.02 & Rejected \\
\hline
\end{tabular}

Source: Own estimation

\section{CONCLUSION}

In the productivity analysis the farmers of Aditmari Upazila attained higher output per hectare $(21208.47 \mathrm{~kg})$ than the farmers of Lalmonirhat Sadar Upazila $(19897.88 \mathrm{~kg})$. In the translog stochastic cost frontier the coefficient of fertilizer price, manure price, per hectare rent of land and per hectare irrigation cost have significantly positive contribution on the increase of cost of production of potato. The mean economic efficiency estimated from translog stochastic cost frontier was $96 \%$. This implies that the cost of production per farm can be reduced by $4 \%$ keeping the output constant.

In the inefficiency effect model there was no significant effect at the aggregate level. But in Aditmari Upazila the coefficient of farm size is positively significant, which means that inefficiency increases with the increase in farm size, while the coefficient of education is negatively significant. This implies that economic inefficiency decreases with the increase in education that means educated farmers are economically more efficient than noneducated or less educated farmers. The variance ratio parameter $\gamma$ associated with the variance in the stochastic frontier is significantly positive for potato production in Aditmari Upazila. It indicates that there are significant economic inefficiency effects in potato production in Aditmari Upazila.

From the above results no concrete policy implication can be drawn since it was a microstudy covering a very small area of Bangladesh. Nevertheless, the following policy implications can be drawn for the improvement of the productive efficiency of potato farmers on the basis of various findings of the study.

From the analysis it was found that education has a positive influence on productivity and efficiency. That means educated farmers had a positive contribution on production and were economically efficient. Several factors were identified as being responsible for the positive impact of the education variable: (i) Educated farmers used more input than non-educated or less-educated farmers, (ii) Educated farmers adopted advanced technology rapidly than less educated farmers, (iii) Educated farmers had the adequate knowledge about the optimum utilization of resources and manage their inputs in the 
most efficient way. Due to the importance of education the following recommendations can be made from this study: (i) The government should give much emphasis on education to improve and sustain the condition of the necessary human capital for agricultural development (ii) Various training programme for the less educated farmers should be taken by the government.

It was observed that the farmers are economically efficient in producing potato and increase in production may not be possible under the existing technology. In this case, only using improved and advanced technology can increase potato production.

In this study it was found that the farmers in Lalmonirhat Sadar Upazila are economically more efficient than the farmers in Aditmari Upazila. So, the government should give much emphasis on the less efficient region without major investment at least in the short run.

\section{REFERENCES}

Battese, G. E. and Coelli, T. J. 1992. "Frontier Production Functions, Technical Efficiency and Panel Data: With Application to Paddy Farmers in India", J. Productivity Analysis, 3: 153-169.

Bangladesh Bureau of Statistics, 2008. Yearbook of Agricultural Statistics of Bangladesh, Ministry of Planning, Government of the People's Republic of Bangladesh, Dhaka.

Bravo-Ureta, B. E. and Rieger, L. 1991. "Dairy Farm Efficiency Measurement Using Stochastic Frontiers and Neoclassical Duality", American J. Agril. Econ., 73(2): 421-428.

Coelli, T. J. 1995. "Recent Developments in Frontier Estimation and Efficiency Measurement", The Australian J. Agril. Econ., 39: 219-245.

Coelli, T. J. 1996a. "A Guide to FRONTIER, Version 4.1: A compute programme for Stochastic Frontier Production and Cost Function Estimation", CEPA Working Paper 96/07, Department of Econometrics, University of New England, Armidale, NSW, 2351, Australia.

Farrell, M. J. 1957. "The Measurement of Productive Efficiency," J. Royal Statistical Society, Series A, 120: $253-290$.

Huang. C. J. and Lui, J. T. 1994. “Estimation of a Non-neutral Stochastic Frontier Production Function", J. Productivity Analysis, 4: 171-180.

Kumbhakar, S. C., Ghosh, S. and Mcguckin, J. T. 1991. “A Generalized Production Frontier Approach for Estimating Determination of Inefficiency in U. S. Dairy Farms", J. Business and Economic Statistics, 9: 279-286.

Parikh, A., Ali, F. and Shah, M. K. 1995. "Measurement of Economic Efficiency in Pakistani Agriculture", American J. Agril. Econ., 77(3): 675-685.

Rahman, K. M. M. 2002. "Measuring Efficiency of Producing Rice in Bangladesh : A Stochastic Frontier Analysis," Wissenschaftsverlag Vauk Kiel KG, Germany, ISBN 3-8175-0357-1.

Reifschneider, D. and Stevenson, R. 1991. "Systematic Departures from the Frontier: A Framework for the Analysis of Firm Inefficiency", Intl. Econ. Review, 32: 715-725.

Stevenson, R. E. 1980. "Likelihood Functions for Generalised Stochastic Frontier estimation", J. Econometrics, 57-66.

Thompson, H. C. and Kelly, W. C. 1957. Vegetable Crops, McGraw Hill Book Company Inc., New York. 